Scientific Journal of Hamadan Nursing \& Midwifery Faculty - ISSN 2008-2819

\title{
The Correlation of Quality of Life With Social Support for Kidney Transplant Patients
}

\author{
Afsar Omidi ${ }^{1}$, Hossein Gholinezhad ${ }^{2}$, Masoud Khodaveisi ${ }^{3^{*}}$, Ghodratollah Roshanaei ${ }^{4}$
}

1. Instructor, Chronic Diseases (Home Care) Research Center Hamadan University of Medical Sciences, Hamadam, Iran

2. Graduate in Community Health Nursing, Department of Community Health Nursing, Faculty of Nursing and Midwifery, Student Research Committee, Hamadan University of Medical Sciences, Hamadam, Iran

3. Associate Professor, Chronic Diseases (Home Care) Research Center, Hamadan University of Medical Sciences, Hamadam, Iran

4. Associate Professor, Department of Biostatistics, School of Health, Hamadan University of Medical Sciences, Hamadan, Iran

\begin{tabular}{lc}
\hline \multicolumn{2}{c}{ Article Info } \\
\hline & \\
Received: & $2017 / 07 / 19$ \\
Accepted: & $2017 / 10 / 7$ \\
Published Online: & $2017 / 10 / 25$
\end{tabular}

DOI:

10.30699/sjhnmf.26.a5.341

Original Article

Use your device to scan and read the article online



\section{Abstract}

Introduction: Kidney transplantation is the best treatment in end stage renal failure. Quality of life is an important factor in the survival of kidney transplants and these patients need social support to improve their quality of life. Therefore, the present study was conducted to determine the correlation between quality of life and social support in patients with renal transplantation.

Methods: This descriptive cross-sectional study was a correlation study performed on 166 patients with kidney transplantation referred to the renal transplantation clinic of Shahid Beheshti Medical Center in Hamadan in the winter of 2016. Convenient sampling method was used. Before the completion of the questionnaires, an informed consent was obtained and researcher was available during completion of questioners. Data was collected using demographic characteristics, Lapazis Quality of Life (KTQ-25) and Social Wax Support questionnaires. Data was analyzed by SPSS24 using Kolmogorov-Smirnov, Mann-Whitney, Kruskal-Wallis and Spearman non-parametric tests.

Results: The mean score of total quality of life was $2.79 \pm 0.99$. The mean score of quality of life in the area of physical symptoms was higher than other areas (3.67) and the total score of social support was $69.91 \pm 7.58 .80 .7 \%$ of patients had an average quality of life. the mean score of social support in the family was more than other areas (25.61\%). 57.2\% of patients had a good level of social support. Social support had a direct and significant association with quality of life of patients $(\mathrm{r}=$ $0.371, P<0.05)$.

Conclusion: There was a direct and significant association between quality of life and the level of social support in patients with renal transplantation. Therefore, it is necessary to pay special attention to the role of families in increasing the quality of life of patients and consequently, their health promotion. Health care personnel can consider the quality and quantity of support needs of patients by mobilizing support resources in the community and family participation to improve the quality of life of patients and as a low-cost and effective way to improve their quality of life.

Keywords: Quality of Life, Social Support, Kidney Transplantation
Corresponding Information

Masoud Khodaveisi, Associate Professor, Chronic Diseases (Home Care) Research Center, Hamadan University of Medical Sciences, Hamadam, Iran. Email: khodaveisi@umsha.ac.ir

Copyright (C) 2018, Sci J Hamadan Nurs Midwifery Fac. This is an open-access article distributed under the terms of the Creative Commons Attribution-noncommercial 4.0 International License which permits copy and redistribute the material just in noncommercial usages, provided the original work is properly cited. How to Cite This Article:

Omidi A, Gholinezhad H, Khodaveisi M, Roshanaei G. The Correlation of Quality of Life With Social Support for Kidney Transplant Patients. Sci J Hamadan Nurs Midwifery Fac. 2018; 26 (5): 341-348 
مجله علمى دانشكدة هرستارى و مامايى همدان - شايا الكترونيك: 19

مقاله يزوهشى

همبستخَى كيفيت زندگى با حمايت اجتماعى در بيماران غييوند كليه






علوم يزشكى همدان، همدان، ايران







افزايش نارسايى مزمن كليوى (V درصد) رو به افزايش است

























مقدمه نارسايى مزمن كليوى مرحلة آخر اختلال كليوى






















تمايل داشته باشند و بهجز يِيوند كليه رييوند ديخرى نداشته










مطالعه بودند.

حجم نمونه با استفاده از فرمولهاى

$$
n=\frac{\left(Z_{1-\frac{3}{2}}+Z_{1-\beta}\right)^{2}}{(C r)^{2}}+3, \quad C r=0.5 \ln \frac{1+r}{1-r}
$$

و با در نظر گرفتن ضريب همبستخى و آ/ • و خطاى نوع اول



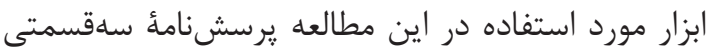














سكونت و تعداد دفعات ييوند بود.

قسمت دوم، يرسشنامة كيفيت زندگى Lapazis






























شده است [IV]

قسمت سوم، يرسشنامهُ حمايت اجتماعى واكس بود













دورة צY شمارة له - آذر و دى Vو I I
اين موضوع كه برخى از بيماران، نسبت به ديخر بيماران،













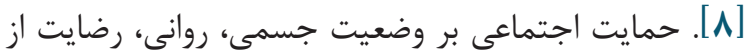








و ديخر افراد تعبير مى شود إنى [11]















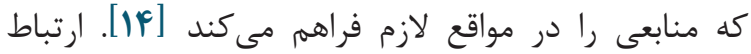





























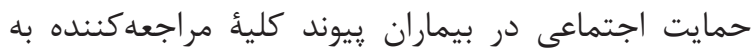



\section{روش كار}

اين مطالعهُ توصيفى ـ مقطعى از نوع همبستتى است



















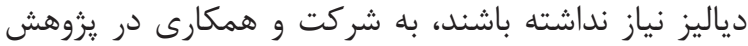






ميانكَين سنى شركت كنند كان در يزوهش


درصد) بودند.

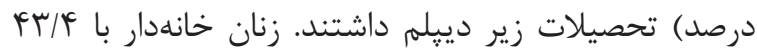













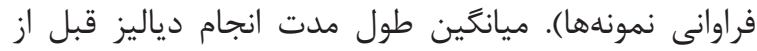

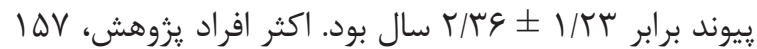







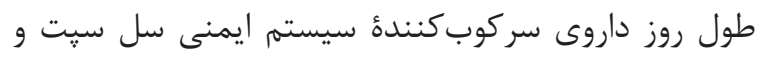

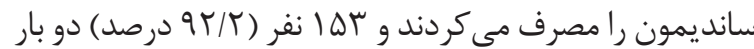











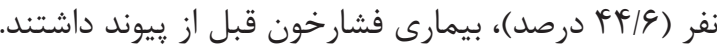

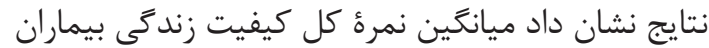















. $(P>\cdot / \cdot \Delta)$









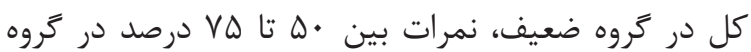

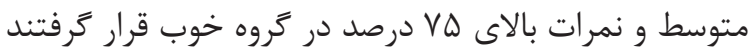

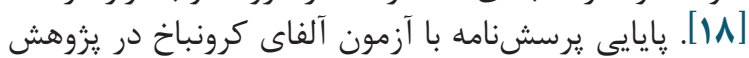
Khabaz

[19]

بِ إز تصويب طرح و كسب اجازه كتبى از مسئولان

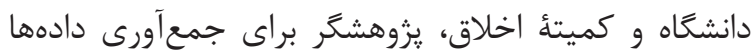







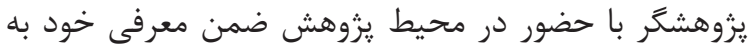

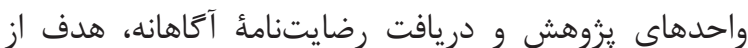



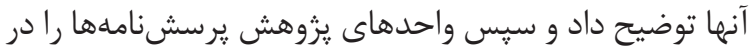
محيطى آرام با حضور يزوهشكر تكميل كردند.




















كرفته شد.

جدول ا. ميانكَين و انحرافمعيار نمرهٔ كيفيت زندكى و حيطههاى آن در افراد يزوهش

\begin{tabular}{|c|c|c|}
\hline (حداكثر - حداقل) نمره & ميانكين و انحرافمعيار & حيطههاى كيفيت زندكى \\
\hline$(1-V)$ & $r / 94 \pm \cdot / 99$ & كيفيت زندگى (كل) \\
\hline$(1 / 1 \vee-V)$ & $r / 9 V \pm 1 / 1 r$ & علائم جسمى \\
\hline$(1-V)$ & $r / 9 \cdot \pm 1 / r \varepsilon$ & خستخى \\
\hline$(1-V)$ & $r / \Delta \cdot \pm 1 / F \Delta$ & ترس/ نبود اطمينان \\
\hline$(1-8 / \vee \Delta)$ & $1 / v \Delta \pm 1 / \cdots$ & ظاهر \\
\hline$(1-V)$ & $\Gamma / \Delta \cdot \pm 1 / \Gamma \Delta$ & عاطفى \\
\hline
\end{tabular}


بين كيفيت زندى بـ با حمايت اجتماعى در بيماران بان بيوند

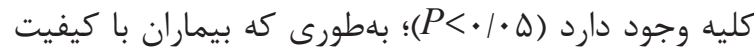

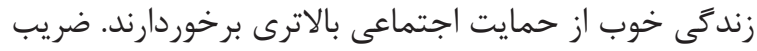







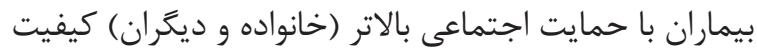



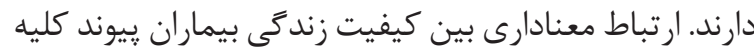



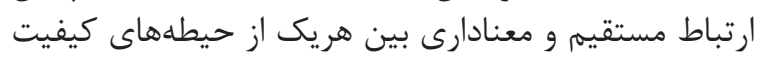




كل حمايت اجتماعى بيماران پيوند كليه



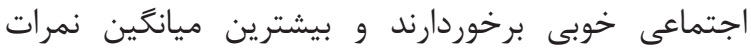


اجتماعى خانواده است (جدول (؟).

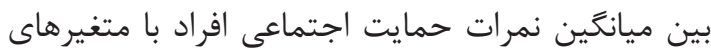



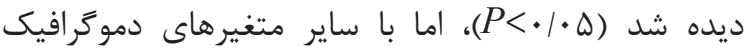





\begin{tabular}{|c|c|c|}
\hline (حداكثر - حداقل) & ميانتين و انحرافمعيار & حيطه هاى حمايت اجتماعى \\
\hline$(q \cdot-\Delta \cdot)$ & $99 / 9) \pm \mathrm{V} / \Delta \wedge$ & حمايت اجتماعى (كل) \\
\hline$(19-M T)$ & $r \Delta|q| \pm r / \mid \varphi$ & خانواده \\
\hline$(\mid F-r \Lambda)$ & $r \cdot / 11 \pm r / 4 V$ & دوستان \\
\hline (I) & $r F / 19 \pm r / 9 r$ & ديخران \\
\hline
\end{tabular}

جدول س. همبستتى كيفيت زندگى با حمايت اجتماعى در افراد مطالعه

\begin{tabular}{|c|c|c|}
\hline \multicolumn{2}{|c|}{ كيفيت زندكى } & \multirow{2}{*}{ حيطه هاى حمايت اجتماعى } \\
\hline$P$-value & ضر يب همبستغى (r) & \\
\hline$P<\cdot / \cdot \Delta$ & $\Leftrightarrow 9 / 9) \pm V / \Delta \Lambda$ & حمايت اجتماعى كل \\
\hline$P<\cdot / \cdot \Delta$ & $\cdot 1909$ & خانواده \\
\hline$P>\cdot / \cdot \Delta$ & $-\cdot|| f \mid$ &  \\
\hline$P<\cdot / \cdot \Delta$ & 每 & ديكران \\
\hline$P$-value & ضر يب همبستغَى (r) حمايس & حيطههاى كيفيت زندىى \\
\hline$P<\cdot / \cdot \Delta$ & $\cdot|r V|$ & كيفيت زندگى كل \\
\hline$P<\cdot / \cdot \Delta$ & $\cdot \pi \cdot \Delta$ & حيطؤ ترس/ عدم اطمينان \\
\hline$P<\cdot / \cdot \Delta$ & $\cdot / r 91$ & حيطة ظاهر \\
\hline$P<\cdot / \cdot \Delta$ &  & حيطؤ جسمى \\
\hline$P<\cdot / \cdot \Delta$ & $\cdot|M T|$ & حيطة خستكى \\
\hline$P<\cdot / \cdot \Delta$ & $\cdot r \cdot r$ & حيطهُ عاطفى \\
\hline
\end{tabular}

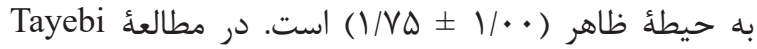



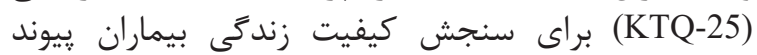



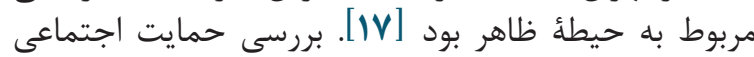
دريافتشده در بيماران קيوند كليه نشان مىدهد كماني كه اكثر

بررسى كيفيت زندگى بيماران بيوند كليه نشان داد



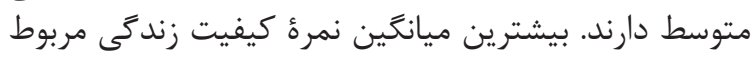
به حيطة فيزيكى (T) 








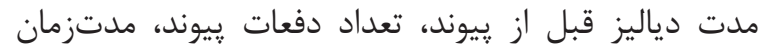

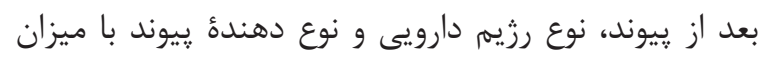

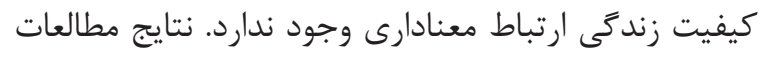

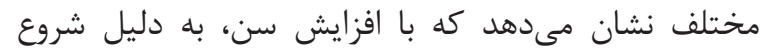

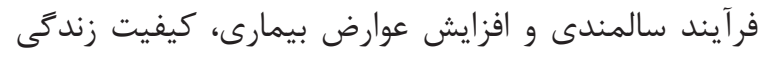

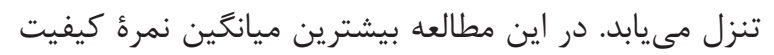

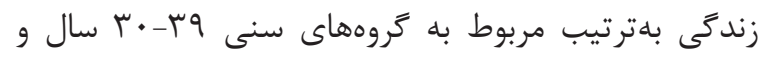

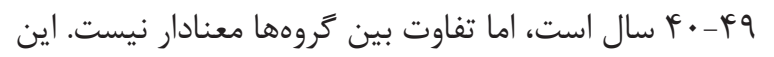

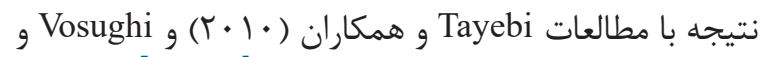
. Movahedpour

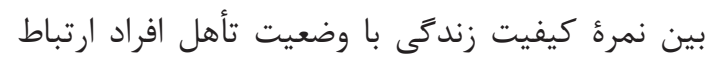

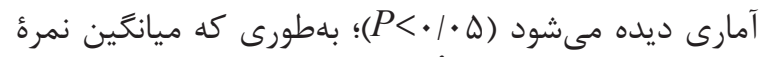

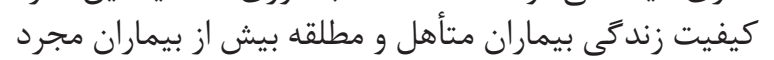








Heydari و و همكاران

(Y.・人)

\section{نتيجه}

نتايج اين تحقيق نشان داد كه بيشتر افراد مطالعه از



























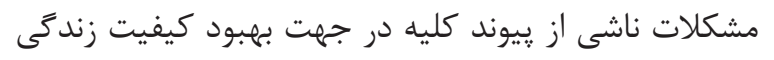





مقالة حاضر بخشى از باياننامهٔ مقطع كارشناسىارشد،



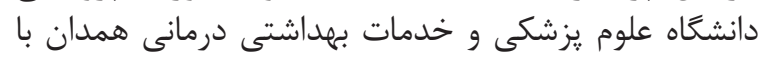




مجوز از اساتيد، معاونت يزوهشى دانشكاه علوم يزشكى همدان،







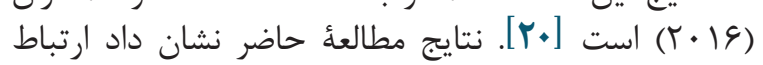

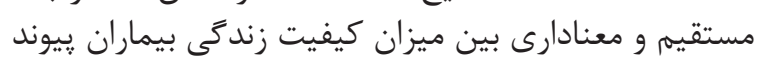

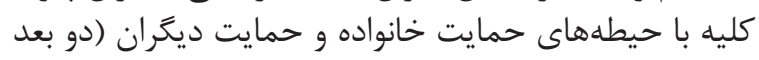

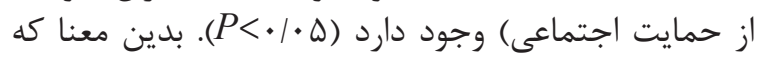

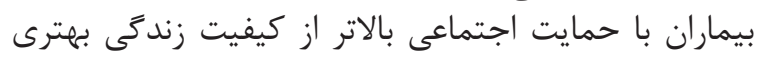

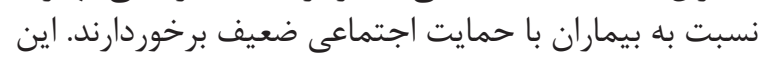

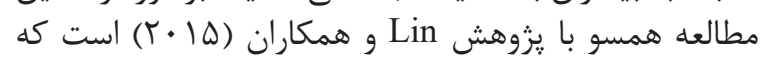

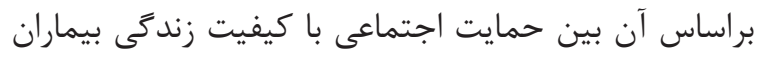

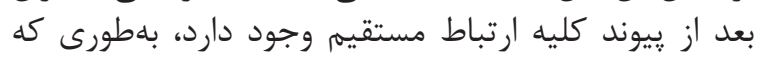

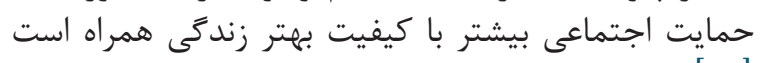

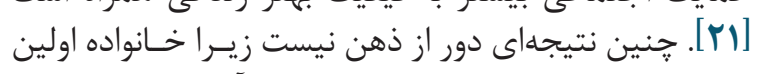

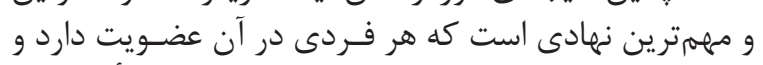

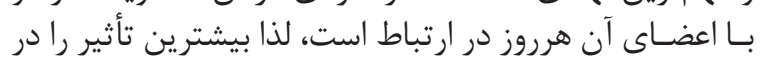









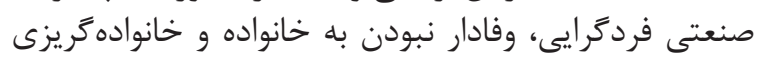



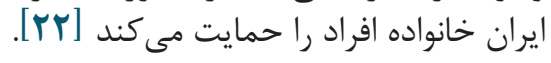







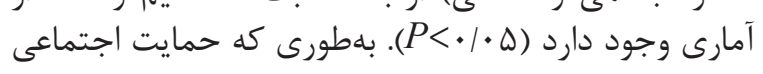



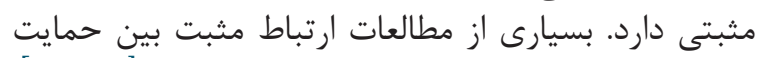

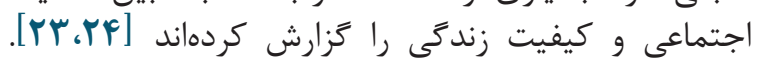
Beattie





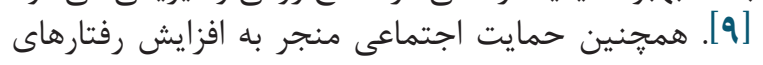

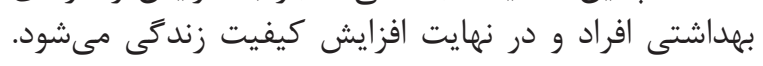









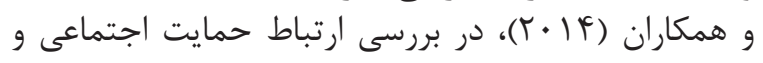





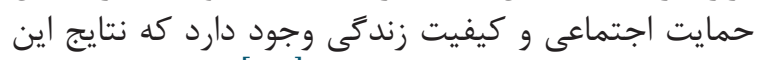



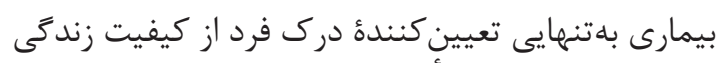



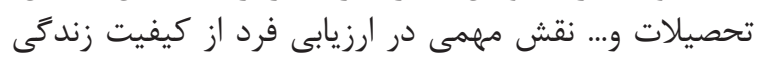








$$
\begin{aligned}
& \text { تعارض منافع } \\
& \text { ميان نويسندًان اين مقاله تعارض منافعى وجود ندارد. }
\end{aligned}
$$

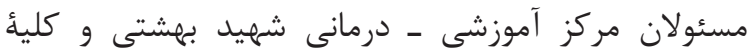

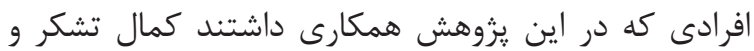

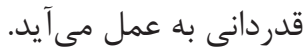

\section{References}

1. Mistretta A, Veroux M, Grosso G, Contarino F, Biondi M, Giuffrida G. Role of socioeconomic conditions on outcome in kidney transplant recipients. Transplantation proceedings. 2009. https:// doi.org/10.1016/j.transproceed.2009.03.017 PMID:19460506

2. Afshar R, Sanavi S, Salimi J. Epidemiology of chronic renal failure in Iran: a four year single center experience. Saudi Journal of Kidney Diseases and Transplantation. 2007;18(2):191. PMID:17496393

3. Bahraminezhad F, Sanaei N, Farokhnezhad P. Transplant Nursing. Tehran: Jameenegar. 2013.

4. Rakrava M, Omranifard V, Ebrahimi A, Atapour A, Mortazavi M. relationship between quality of life, general health and dysfunctional attitudes in kidney transplant patients. Journal of Research in Behavioural Sciences. 2015;12(4):510-9.

5. Shrestha A, Vallance C, McKane W, Shrestha B, Raftery A, editors. Quality of life of living kidney donors: a single-center experience. Transplantation proceedings. 2008.

6. Unruh M, Weisbord S, Kimmel P. Psychosocial factors in patients with chronic kidney disease: Health-related quality of life in nephrology research and clinical practice. Seminars in dialysis. 2005:Wiley Online Library.

7. Ferrans CE, Powers MJ. Quality of life of hemodialysis patients. Anna Journal. 1993;20(5):57581.575-81.

8. Shahraki M, Shahraki T, Gargari B, Ramroudi N. Central Obesity and Coronary Heart Disease Risk Factors in Referral Outpatients to Zahedan Cardiology Clinic, Iran. International journal of high risk behaviors \& addiction. 2012;1(2):79-83.https://doi.org/10.5812/ijhrba.4275 PMID:24971238 PMCID:PMC4070110

9. Beattie S, Lebel S, Tay J. The influence of social support on hematopoietic stem cell transplantation survival: a systematic review of literature. PloS one. 2013;8(4):e61586. https://doi.org/10.1371/ journal.pone.0061586 PMID:23637862

10. Aalto A-M, Uutela A. Glycemic control, selfcare behaviors, and psychosocial factors among insulin treated diabetics: a test of an extended health belief model. International journal of behavioral medicine. 1997;4(3):191-214. https://doi.org/10.1207/s15327558ijbm0403 1

\section{PMID: $\underline{16250728}$}

11. Von Kanel R. Psychosocial stress and cardiovascular risk: current opinion. Swiss Med Wkly. 2012;142:w13502. PMid:22271452

12. Hwang S, Kirst M, Chiu S, Tolomiczenko G, Kiss A, Cowan L. Multidimensional social support and the health of homeless individuals. Journal of Urban Health. 2009;86(5):791-803. https://doi. org/10.1007/s11524-009-9388-x PMid:19629703 PMCid:PMC2729873

13. Taher M, Abredari H, Karimy M, Rahmati M, Atarod M, Azaepira H. The Assessment of Social Support and Self-Care Requisites for Preventing Diabetic Foot Ulcer in Diabetic Foot Patients. Journal of Education And Community Health. 2016;2(4):34-40. https://doi.org/10.21859/jech$\underline{02045}$

14. Findler L, Jacoby A, Gabis L. Subjective happiness among mothers of children with disabilities: The role of stress, attachment, guilt and social support. Research in developmental disabilities. 2016;55:44-54. https://doi.org/10.1016/j. ridd.2016.03.006 PMid:27037989

15. Namdar H. Compliance in Patients with Schizophrenia. Iran Psychiatry Clinical Psychol 2005;4(43):120.

16. Pus N. Administrative guide to the kidney transplantquestionnaire. London: Robarts Research Institute. 1999.

17. Tayebi A, Raiesifar A, Ebadi A, Eynollahi B, Rafiyan Z. Review the renal transplantation patients' quality of life by using kidney transplantation questionnaire (KTQ-25). Iranian Journal of Critical care Nursing. 2010;3(3):125-8.

18. Vaux A, Riedel S, Stewart D. Modes of social support: The social support behaviors (SS-B) scale. American Journal of Community Psychology. 1987;15(2):209-32. https://doi.org/10.1007/ $\underline{\mathrm{BF} 00919279}$

19. Khabaz M, Behjati Z, Naseri M. Relationship between Social Support and Coping Styles and Resiliency in Adolescents. Journal of Applied Psychology 2012;5(4):108-23.

20. Mikula P, Nagyova I, Krokavcova M, Vitkova M, Rosenberger J, Szilasiova J. Self-esteem, social participation, and quality of life in patients with multiple sclerosis. Journal of health psychology. 2016. 
21. Lin X, Shang Y, Teng S, Liu H, Han L. Relationship between Perceived Social Support and Quality of Life among Kidney Transplant Recipients. GSTF Journal of Nursing and Health Care. 2015;3(1).

22. Osterberg L, Blaschke T. Adherence to medication. New England Journal of Medicine. 2005;353(5):487-97.https://doi.org/10.1056/NEJMra050100 PMid: 16079372

23. Bennett S, Perkins S, Lane K, Deer M, Brater D, Murray M. Social support and health-related quality of life in chronic heart failure patients. Quality of life research. 2001;10(8):671-82.https://doi. org/10.1023/A:1013815825500 PMID: 11871588

24. Helgeson V. Social support and quality of life. Quality of life research. 2003;12(1):25-31. https://doi.org/10.1023/A:1023509117524 PMid: 12803308

25. Sanne B, Mykletun A, Dahl A, Moen B, Tell G. Testing the job demand-control-support model with anxiety and depression as outcomes: The Hordaland Health Study. Occupational medicine. 2005;55(6):463-73. https://doi.org/10.1093/occmed/kqi071 PMID:15845554

26. Medellín Olaya J, Carrillo González G. Per-

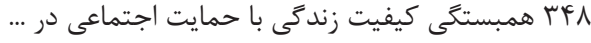

ceived social support and quality of life of people with chronic kidney disease undergoing renal transplantation. Avances en Enfermería. 2014;32(2):206-16.https://doi.org/10.15446/ av.enferm.v32n2.46099

27. Vosughi M, Movahedpour A. Comparison quality of life between hemodialysis and transplant patient in Ardabil medical centers. Ardabil Univ Med Sci J. 2009;9(2):171-9.

28. Taher M, Abredari H, Karimy M, Abedi A, Shamsizadeh M. The Relation Between Social Support and Adherence to the [Treatment of Hypertension]. J Edu Commun Health. 2014;1(3):63-9. https://doi.org/10.20286/jech-010348

29. Woodward E, Pantalone D. The role of so social support and negative affect in medication adherence for HIV-infected men who have sex with men. J Assoc Nurses AIDS Care. 2012;23(5): 388 96. https://doi.org/10.1016/j.jana.2011.09.004 PMID:22209470 PMCID:PMC3319472

30. Heydari S, Noori Tajer M, Hoseini F, Inanloo M, Golgiri F, Shirazi F. Family Support and Diabetic Type-2 Glycemic Control. Salmand. 2008;3(2):573-80 